\title{
The Effect of Ego Depletion on Arousal of Elite Iranian Swimmers
}

\author{
${ }^{1}$ Saber Mehri", ${ }^{1}$ Zahra Pooraghaei Ardekani, ${ }^{2}$ Mohammad Khabiri \\ ${ }^{1}$ Department of Sport Psychology, Faculty of Sport Sciences, Allameh Tabataba'i University, Tehran, Iran. ${ }^{2}$ Department of \\ Sport Management, Faculty of Sport Sciences, Tehran University, Tehran, Iran.
}

\begin{abstract}
The purpose of the present study is to examine the effects of ego depletion on arousal of elite Iranian swimmers. The sample comprised elite swimmers invited to the national swimming team as focused and semi focused (36 subjects). The research design was as pre- and post-test - in two phases of ego depletion conditions and the lack of ego depletion conditions in two experimental and control groups. The arousal was measured by a biofeedback device, and Stroop test was conducted in order to create ego depletion conditions. The results showed that ego depletion had no effect on the arousal of elite swimmers. In other words, the results showed that depletion of strength sources of self-control had no effect on elite swimmers' arousal. The findings - based on the theory of community facilities and the self-control model-showed that arousal is actually an accelerator and moderator of using self-control sources; it is not a factor using self-control sources and, also, ego depletion is not involved in arousal changes.
\end{abstract}

KEY WORDS: Self-Control, Self-Regulation, Ego Depletion, Arousal.

\section{INTRODUCTION}

These days, many researchers examine psychological aspects and factors affecting championship. The skill of self-regulation in sports is a good reason to justify the athletes' function, facing difficulties like professional racing conditions (1). Self-regulation refers to any human effort to change emotions, thoughts and behaviours in a manner consistent with demands (2). Self-regulation with resistance to improper motives relies on proper behaviour and facilitates desirable conditions (3). Behaviours resist other behaviours against being neglectedfor example, the need to get free of a painful stimulus is usually more difficult than ignoring it (4).

*. Corresponding Author:

Saber Mehri

E-mail: saber_neverstop@yahoo.com
In order to carry out perceptual-motor activities, removing annoying stimuli like the sound of the audience and irrelevant thoughts is essential (5). Most people have trouble in adjusting their focus under high-pressure conditions that could have a negative impact on perceptual-motor function (6). Several studies have shown that higher levels of anxiety are associated with dysfunction in different perceptual-motor tasks: for example, in basketball free throws (7). In addition, according to attentional control theory, if anxiety is higher, more irrelevant stimuli and disturbing thoughts are received (8). 
The self-control model states that all acts of self-control stem from the same source with specified capacity. Based on the model of selfcontrol strength, a self-control primary act can remove self-control strength of a person so that it is not immediately reconstructed. This period is called ego depletion and then self-control measures cannot be effective as before on the implementation of functions, and impair the function (3).

This theoretical assumption has been given empirical support, and studies have shown that those with ego depletion in the case of arousal, emotional, and attention regulation or complex cognitive operations, get worse functions. Most of the functions require will, such as decisionmaking, choice, accountability, innovation and behavioural inhibition, planning and doing them (9).

The ability to control acts as an important part of everyday life. Waking up and attending work on time, instead of watching TV and clubbing, are examples of the use of self-control. If we can regulate actions, it is very difficult. For example, overeating can have detrimental consequences for health. What is the reason for this type of problem? The strength self-control model acknowledges that acts require selfcontrol to reduce sources, so that in practice, self-control strength is reduced (10).

Removal of self-control sources is called ego depletion (2). In other words, cognitive resources can be reduced by using a process called ego depletion (11). Ego depletion refers to the idea that self-control and will are from a limited mental source (10). Also, when mental activity energy is low, self-control is often impaired, and this case is called ego depletion. In particular, experiencing what is called ego depletion impairs the ability to do the next task. Tasks that require self-control can have a preventive effect on the next self-control task, even if tasks are seemingly unrelated to each other; for example, adjusting a person's emotions could impair a function, like solving a difficult puzzle, squeezing a rubber ring, keeping in mind mental representation in working memory, or reading the colour of words printed in Stroop test (4, 9, $12,13)$. Self-control plays an important role in personal and interpersonal function. Ego depletion is an important subject in experimental psychology, in particular, social psychology, because this phenomenon is a mechanism that aids the understanding of human control processes (14).

Willingly preventing something or changing the response can be considered an act of selfcontrol (3). The self-control strength model (13) proposes that all acts of self-control by the same sources have metaphorical strength with limited capacity. Regarding the strength model, a primary act of self-control can remove selfcontrol strength, but sometimes this is not filled immediately. During this period of ego depletion, self-control acts cannot be performed effectively as before, leading to dysfunction of self-control measures (10).

Arousal is referred to as a set of emotions, anxieties, attention, concentration, stress and motivation. Martens said arousal is a state referred to as the physiological, mental and behavioural activation of the body. Elizabeth Duffy - the most famous theorist of arousaldefined the term as releasing the potential energy that is stored in the tissues of the organism and manifested in the activity or response. Movement behaviourists used motivation as a theoretical basis for arousal structure. In particular, Sage defined arousal as an energy function that shows the intensity of motivation and conducts behaviour towards a specific goal. Magill (1992) considered arousal to be synonymous with activation. He stated that a person's motivation is, in fact, motivating or enabling that person to prepare him for the task (15). Several theories have been expressed about arousal, such as the inverted $U$ theory, which holds that at low levels of arousal, function will be lower than the average, and the athlete in the state is not mentally ready. When arousal increases, function is increased up to the optimized point that gives the best function. But a further increase in arousal causes deterioration in the function. So, this theory is shown with an inverted $U$, reflecting a high function at optimized levels of arousal and lower function at low or high arousal levels (16).

Evidence has shown that ego depletion does not directly affect arousal, but will reduce the boredom threshold and boredom will reduce arousal (9). According to the above, the question is that in swimming, that desirable function 
requires attention control, self-control and desirable arousal. Does ego depletion affect arousal, or does it not?

\section{MATERIALS AND METHODS}

Participants. The sample comprised focused and semi-focused elite swimmers invited to the national swimming team (36 subjects). Research design was as pre- and post-test, in two phases of ego depletion conditions and lack of ego depletion conditions in the two experimental $(n=18)$ and control groups $(n=18)$.

Research Design. For the pre-test, a baseline of subjects was determined as the pretest of arousal component by a biofeedback device. In this study, arousal was determined through skin resistance by a biofeedback device (model RT-935). The mechanism for determining arousal is a way in which changes in electrical conduct or resistance of the skin under sweat glands in controlling autonomous nervous system were recorded and provided perfect aural feedback. The validity of this device was confirmed by the manufacturer at a desirable level. Its reliability-compared with similar foreign devices - was .86 (15).

After determining the basic level of arousal, using Stroop Test, the ego depletion cognitive state was made for a person and immediately after Stroop efforts; completion using biofeedback subjects' arousal was recorded. In Stroop Test, red, blue, green and yellow words were written with colored inks, so that it is tested whether or not the text color is consistent with the written word. Subjects should immediately answer by the color and not the word, by pressing the keyboard that colors were in accordance with the answer. The Stroop test used in this study was the Persian version of Stroop software produced in Sina BehavioralCognitive Sciences Research Institute, which carried out research on the test to show proper reliability and validity of this test in adults. By the retest method, reliability of the test was in the range of .6 to .97 (17). To run this test, subjects - after sitting in front of the computer screen faced 96 randomized efforts (including 48 consistent and 48 inconsistent stimuli) so that they should detect ink colors of the words. This test time was 4 minutes, and software calculated subjects' scores individually for consistent and inconsistent stimuli. Immediately after performing the Stroop test, post-test was held. Generally reduced in ego depletion research Stroop test is used.

Data Analysis. To analyze the data, both descriptive and inferential statistics were used. To classify and regulate raw data, average, variations' range, and standard deviation were used, and in inferential statistics, KolmogorovSmirnov test and Levene statistics were used to examine homogeneity of variances, and ANCOVA test was used to determine differences between the study groups. It should be noted that all tests were performed at $p<0.05$. All statistical calculations of each of the hypotheses were carried out using software SPSS.

\section{RESULTS}

In order to meet assumptions of parametric statistics, KS test was used to determine the normality of the distribution of data. The results are presented in Table 1. As seen, all data categories studied have a normal distribution $(\mathrm{P}>0.05)$.

Table 1. The results of KS test to examine normality of data distribution

\begin{tabular}{cccc}
\hline Variable & $\mathbf{t}$ & $\mathbf{d f}$ & $\mathbf{p}$ \\
\hline Arousal & 0.086 & 36 & 0.2 \\
\hline
\end{tabular}

Then, in order to examine the homogeneity of variances, Levene's statistical test was used. Table 2 shows the results of Levene's test on homogeneity of variances.

Table 2. The results of Levene's test on homogeneity of variances

\begin{tabular}{ccccc}
\hline Variable & $\mathbf{f}$ & $\begin{array}{c}\text { Intra- } \\
\text { group df }\end{array}$ & $\begin{array}{c}\text { Intra- } \\
\text { group df }\end{array}$ & $\mathbf{p}$ \\
\hline Arousal & 0.175 & 1 & 34 & 0.679 \\
\hline
\end{tabular}

As can be seen, the test significance level was obtained equal to 0.679 . So, based on the decision principle, homogeneity of variances has been studied in groups.

Also, the results of analysis of one-way covariance showed that to test the main hypothesis of the study, after adjusting pre-test 
scores in arousal variable, no significant difference was found between the scores of posttest in both control and experimental groups.
Table 3 indicates the results of one-way analysis of covariance of arousal variable.

Table 3. The results of one-way analysis of covariance of arousal variable

\begin{tabular}{cccccc}
\hline & Mean Square & df & $\mathrm{F}$ & $\mathrm{P}$ & $\mathrm{p} \eta^{2}$ \\
\hline Group effect & 4.372 & 1 & 0.003 & 0.955 & 0.001 \\
Pre-test & 176668.436 & 1 & 132.490 & 0.001 & 0.801 \\
Error & 1333.448 & 33 & & & \\
Total & & 36 & & & \\
\hline
\end{tabular}

\section{DISCUSSION AND CONCLUSION}

The purpose of the present study was to examine the effect of ego depletion on arousal of Iran's elite swimmers. Data analysis showed that ego depletion had no effect on the arousal of elite Iranian swimmers. Willingly preventing something or changing the response can be considered an act of self-control-and research has shown that a primary act of self-control can remove self-control strength of a person for a while, so that it is not immediately reconstructed, and ego depletion occurs (2). Then, self-control acts cannot be effectively carried out as before, and this leads to dysfunction in most of the self-control measures (1).

The scientific hypothesis has received considerable empirical support. Several studies have shown that ego depletion of participants in actions that need arousal, emotion, and attention regulation, or complex cognitive operations, had the worst performance. In a recent meta-analysis study, Hagger et al. (2010) found that ego depletion affected the exercise of the next acts of self-control. There are several other theoretical considerations in respect of the basic processes (11). By challenging boredom, it is argued that observing the effects of ego depletion is justified by allocating sources, motivation and changing attention, calculating costs/profits and/or mental theory about will limitation (18).

Baumeister et al. (1998) examined ego depletion of self-control sources in the face of temptation that someone who was forced to eat radishes instead of sweet chocolate much earlier than those who had not been forced to implement, refused an unsolvable puzzle. In other words, to use self-control sources against eating sweets, self-control sources were depleted in solving the puzzle (10). They showed that the reason they get out of the unsolvable puzzle sooner was reduced self-control sources; arousal of individuals in the control and experimental groups had no significant difference (10). Also, Osgood (2015) examined how ego depletion increased boredom and reduced direct attention (9). He found that ego depletion does not directly affect arousal, but reduced the threshold of boredom, and arousal is reduced (9). According to the findings, perhaps no change in the levels of arousal means that change in arousal are changes in sources of self-control and change in arousal are not related to selfcontrol sources. The likely observed reduced function under depletion conditions was not due to reduced arousal. Many researchers have studied the relationship between arousal and performance, and theories have been developed. Based on the theory of inverted U, low levels of arousal will cause a function lower than the average, and the athlete in this case will not be mentally ready. When arousal is increased, the function is increased up to the optimized point, which gives the best result. But a further increase in arousal causes function deterioration. So, this theory is shown with an inverted U, reflecting high function at an optimized level of arousal and low function at low or very high levels of arousal.

The relationship between ego depletion and arousal requires deeper analysis. Social Facilitation Theory (1965) states that the presence of others causes arousal in a performer, damaging function in difficult tasks not learned good yet, but helps the implementation of good learned tasks. Subramaniam (2011) stated this 
theory in connection with ego depletion, as arousal of simple tasks causes insignificant use of self-control sources and vice versa, and arousal of difficult tasks causes significant use of self-control sources; in fact, arousal is an accelerator and moderator of self-control sources use not a factor of self-control sources use. Ego depletion has no role in arousal changes, too (19).

\section{APPLICABLE REMARKS}

- Motivational techniques such as listening to music or watching clips that are used to enhance or maintain arousal, don't lead to depleted selfcontrol resources.

\section{REFERENCES}

1. Barkhoff H, Heiby EM, Pagano IS. Self-Regulation Skills of a Competitor Type vs. a Training Champion Athlete in Artistic Roller Skating: A Season Long Case Study in Elite Sport Competitions. Athletic Insight: The Online Journal of Sport Psychology. 2007;9(2):43-57.

2. Baumeister RF, Vohs KD, Tice DM. The Strength Model of Self-Control. Current Directions in Psychological Science. 2007;16(6):351-5.

3. Baumeister RF, Heatherton TF, Tice DM. Losing control: How and why people fail at self-regulation. San Diego, CA, US: Academic Press; 1994. xi, 307 p.

4. Hull CL. Principles of Behavior: An Introduction to Behavior Theory: Appleton-Century-Crofts; 1943. 422 p.

5. Abernethy B, Maxwell JP, Masters RSW, Kamp JVD, Jackson RC. Attentional Processes in Skill Learning and Expert Performance. Handbook of Sport Psychology: John Wiley \& Sons, Inc.; 2007. p. 245-63.

6. Baumeister RF. Choking under pressure: self-consciousness and paradoxical effects of incentives on skillful performance. Journal of personality and social psychology. 1984;46(3):610-20.

7. Vohs KD, Baumeister RF, Schmeichel BJ. Motivation, personal beliefs, and limited resources all contribute to self-control. Journal of Experimental Social Psychology. 2012;48(4):943-7.

8. Eysenck MW, Derakshan N, Santos R, Calvo MG. Anxiety and cognitive performance: attentional control theory. Emotion. 2007;7(2):336-53.

9. Osgood JM. Acute Cardiovascular Exercise Counteracts the Effect of Ego-Depletion on Attention: How EgoDepletion Increases Boredom and Compromises Directed Attention. International Journal of Psychological Studies. 2015;7(3):85-96.

10. Baumeister RF, Bratslavsky E, Muraven M, Tice DM. Ego depletion: Is the active self a limited resource? Journal of personality and social psychology. 1998;74(5):1252-65.

11. Hagger MS, Wood CW, Stiff C, Chatzisarantis NLD. Self-regulation and self-control in exercise: the strengthenergy model. International Review of Sport and Exercise Psychology. 2010;3(1):62-86.

12. Johns M, Inzlicht M, Schmader T. Stereotype Threat and Executive Resource Depletion: Examining the Influence of Emotion Regulation. Journal of experimental psychology General. 2008;137(4):691-705.

13. Muraven M, Baumeister RF. Self-regulation and depletion of limited resources: does self-control resemble a muscle? Psychological bulletin. 2000;126(2):247-59.

14. Baumeister RF. Yielding to Temptation: Self-Control Failure, Impulsive Purchasing, and Consumer Behavior. Journal of Consumer Research. 2002;28(4):670-6.

15. Esmaeili N. The effect of presence of spectator's arousal on Adaptive reaction time. Tehran, Iran: Allameh Tabataba'i Universtiy; 2015 [Thesis in Farsi].

16. Weinberg RS, Gould D. Foundations of Sport and Exercise Psychology, 6E: Human Kinetics; 2014. 664 p.

17. Pourmohamadi S, Bagheri F. Effectiveness of Mindfulness Training on Automatic Cognitive Processing. Psychological Studies. 2015;11(3):141-59 [Article in Farsi].

18. Englert C, Bertrams A. The effect of ego depletion on sprint start reaction time. J Sport Exerc Psychol. 2014;36(5):506-15.

19. Subramaniam S. Ego Depletion and Delay of Gratification. Statesboro, Georgia: Georgia Southern University; 2011. 